\title{
Fringing Effect Analysis of Parallel Plate Capacitors for Capacitive Power Transfer Application
}

Chen, Xu; Zhang, Zhe; Yu, Shengbao; Zsurzsan, Tiberiu-Gabriel

Published in:

Proceedings of 4th IEEE International Future Energy Electronics Conference

Publication date:

2019

Document Version

Peer reviewed version

Link back to DTU Orbit

Citation (APA):

Chen, X., Zhang, Z., Yu, S., \& Zsurzsan, T-G. (2019). Fringing Effect Analysis of Parallel Plate Capacitors for Capacitive Power Transfer Application. In Proceedings of 4th IEEE International Future Energy Electronics Conference IEEE.

\section{General rights}

Copyright and moral rights for the publications made accessible in the public portal are retained by the authors and/or other copyright owners and it is a condition of accessing publications that users recognise and abide by the legal requirements associated with these rights.

- Users may download and print one copy of any publication from the public portal for the purpose of private study or research.

- You may not further distribute the material or use it for any profit-making activity or commercial gain

- You may freely distribute the URL identifying the publication in the public portal

If you believe that this document breaches copyright please contact us providing details, and we will remove access to the work immediately and investigate your claim. 


\title{
Fringing Effect Analysis of Parallel Plate Capacitors for Capacitive Power Transfer Application
}

\author{
Xu Chen ${ }^{1,2}$, Zhe Zhang ${ }^{1}$, Shengbao $\mathrm{Yu}^{2}$ and Tiberiu-Gabriel Zsurzsan ${ }^{1}$ \\ ${ }^{1}$ Department of Electrical Engineering, Technical University of Denmark, Kgs. Lyngby, Denmark \\ ${ }^{2}$ College of Instrumentation and Electrical Engineering, Jilin University, Changchun, China \\ xuchen@elektro.dtu.dk, zz@elektro.dtu.dk, yushengbao@jlu.edu.cn, tgzsur@elektro.dtu.dk
}

\begin{abstract}
The classical formula of a parallel plate capacitor (PP-Cap) does not take fringing effects into consideration, which assumes that the side length of a PP-Cap is by far larger than the distance between the two plates. However, for capacitive power transfer applications, especially those designed for electric vehicle charging, this assumption no longer holds since the distance can be as large as $150 \mathrm{~mm}$. Based on conformal mapping, the corrected or improved formula of PP-Cap with the consideration of fringing effect can be obtained; nevertheless, some approximations are introduced for the convenience of calculation. By finite element method (FEM) simulation and experimental measurement, this paper investigates the influencing factors of large distance PP-Cap especially in the capacitive power transfer application and thereby the proposed formula with improved accuracy is verified.
\end{abstract}

Keywords - capacitive power transfer, conformal mapping, fringing effect, wireless power transfer

\section{INTRODUCTION}

Capacitive power transfer (CPT) is one of the wireless power transfer methods that using high frequency electric field as the energy transferring medium. Several advantages have been proved from prior arts regarding this method, including tolerance to metal objects [1], cost effective [2] and low standby power loss [3][4]. In a CPT application, wireless couplers between the transmitter and receiver are, typically, two or more parallel plate capacitors (PP-Cap), as shown in Fig.1. Since the relative permittivity of air is only $8.854 \times \mathrm{e}^{-12}$ $\mathrm{F} / \mathrm{m}$, the capacitor plate has to be designed relatively large in order to increase the coupling capacitance.

Some researchers have demonstrated that even with $\mathrm{pF}$ level coupling capacitance, the transferred power can still goes up to $\mathrm{kW}$-level, which makes CPT a possible solution in electric vehicle wireless charging applications [5]-[7]. Several coupler structures including six-plate coupler [5], four-plate coupler [6], [8] and electric field repeater [7] are investigated to increase the coupling coefficient and at the same time avoid the side effect of fringing field. The dimensions of the capacitive couplers reported in [5]-[8] are summarized in Table I, in which the air gap distance ranges are from $150 \mathrm{~mm}$ to $180 \mathrm{~mm}$ and the side length ranges are from $300 \mathrm{~mm}$ to 914 $\mathrm{mm}$, respectively. All of them are targeted for electric vehicle wireless charging applications.

There are several ways to obtain the coupling capacitance of a PP-Cap with given side length $L$ and air gap distance $d$. The simplest one is the calculation of using classical equation i.e. $C_{0}=\varepsilon L^{2} / d$, which assumes the electric field is uniform and also perpendicular to the capacitor electrodes. To increase the

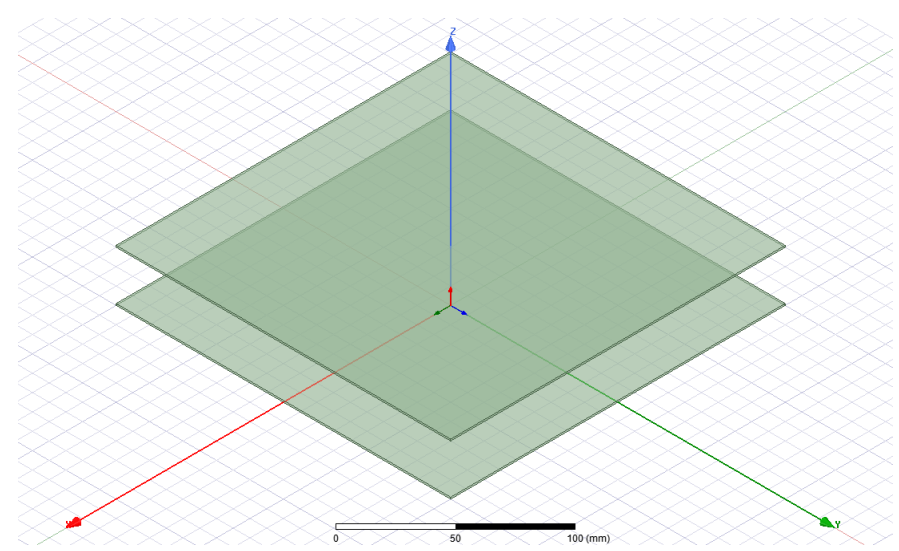

Figure 1. Parallel plate capacitor for wireless power transfer.

TABLE I.

Size AND CAPacitance of Parallel Plate Capacitor in CAPaCitive POWER TRANSFER APPLICATION

\begin{tabular}{|c|c|c|c|c|}
\hline Ref. & $\begin{array}{c}\text { Side length } \\
(\mathbf{m m})\end{array}$ & $\begin{array}{c}\text { Air gap } \\
\text { distance } \\
(\mathbf{m m})\end{array}$ & $\mathbf{r}$ & $\begin{array}{c}\text { Capacitance } \\
\mathbf{( p F )}\end{array}$ \\
\hline$[5]$ & 610 & 150 & 4.07 & 20 \\
\hline$[6]$ & 914 & 150 & 6.09 & 22.6 \\
\hline$[7]$ & 300 & 180 & 1.67 & 16.2 \\
\hline$[8]$ & 500 & 150 & 3.33 & 26.58 \\
\hline
\end{tabular}

calculation accuracy, the fringing field need to be taken into consideration. Fringing effect represents the non-uniform electric fields around the edge of a PP-Cap. When the ratio $r$ (side length $L$ over air gap distance $d, r=L / d$ ) of a PP-Cap is large enough, for example $r>10$, fringing effect can be neglected without seriously affecting the calculation error. However, for PP-Cap in kW-level capacitive power transfer application, $r$ is normally less than 10 according to Table I. To give an accurate calculation result during system design stage, the fringing effect of this category of PP-Cap need to be taken into consideration.

Calculating the fringing effect capacitance is a classical electrostatic field problem. By using conformal mapping, a corrected formula that considering fringing effect can be derived. A number of researchers have reported the calculation results of this problem with similar formula structure and different coefficients [9]-[12]. However, usinconformal mapping needs some assumptions and approximations, which make the calculation result accurate only for a certain range of PP-Cap dimensions. This paper 


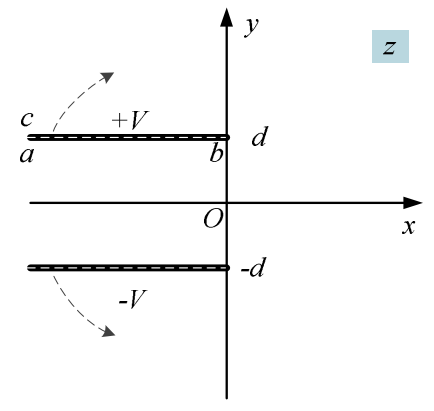

(a)

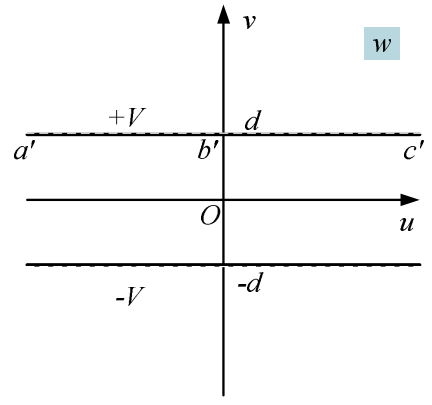

(b)
Figure 2. Conformal mapping of PP-Cap from z-plane to $\omega$-plane.

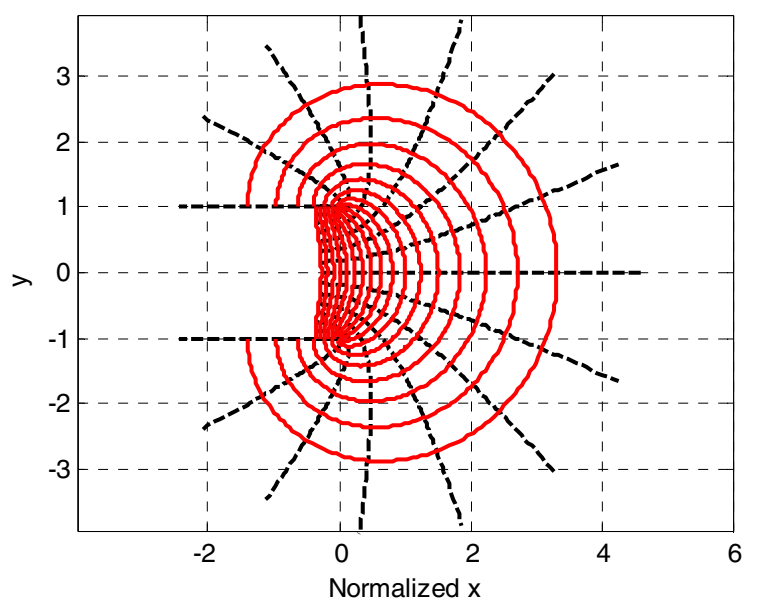

Figure 3. Calculated equipotential line and electric field line around the edge of PP-Cap.

compares the calculation results of these formulas in $\mathrm{kW}$-level CPT applications. Additionally, the finite element method (FEM) can also be used to calculate the coupling capacitance.A comparison of the calculation results, FEM simulation results and measurement results are given with their associated error analyzation. As all the calculation formulas have similar structures, to give an accurate calculation result for PP-Cap in $\mathrm{kW}$-level CPT application, a general formula with coefficients to be defined is proposed in this paper and a new set of coefficients are obtained based on a weighted curve fitting method.

This paper is organized as follows. After this introduction, Section II describes the fringing effect model used to evaluate impacts due to the coupler parameters as well as compare different capacitance formula. Section III provides the FEM simulation and its associated analysis of fringing effect. Section IV combines the results from calculation, simulation and measurement in order to verify the proposed method. Finally, Section V presents our conclusion.

\section{FRINGING EFFECT MODELING}

Fig.2 (a) shows the projection of a PP-Cap on $z$ plane, where line $a b$ is the upper capacitor plate and the lower capacitor is symmetrical to the upper plate about $x$-axis. For conformal mapping, only the fringing effect of the right side of the capacitor is considered. For a given function

$$
\omega=f(z)
$$

that transforms a curve set from $z$-plane to $\omega$-plane, if the local orientation and angles of the curve set preserves during the transformation. This transformation is called conformal mapping.

Define the mapping function as

$$
z=\frac{d}{2 \pi}\left(1+\omega+e^{\omega}\right)
$$

where the PP-Cap is put in the z-plane as shown in Fig. 2(a). Here, we assume that the capacitor plate has infinite length alone the direction perpendicular to the $z$ plane, which ensures that the fringing field is unchanged along this direction. Thus, the $3 \mathrm{D}$ capacitor can be simplified to a $2 \mathrm{D}$ capacitor in $z$ plane. Note that conformal mapping cannot handle 3D problems.

Through the mapping given by equation (2), the upper plate of PP-Cap a-b-c are mapped to $a^{\prime}-b^{\prime}-c^{\prime}$ in $\omega$-plane and so does the lower electrode. The parametric equations between $z$-plane and $\omega$-plane can be solved from (2) as

$$
\begin{gathered}
x=\frac{d}{2 \pi}\left(1+u+e^{u} \cos v\right) \\
y=\frac{d}{2 \pi}\left(v+e^{u} \sin v\right)
\end{gathered}
$$

where $v \in(-\pi ; \pi)$ and $u \in(-\infty ; \infty)$.

The whole $z$-plane is now compressed to a banded area in $\omega$-plane by cutting from the ray line where the capacitor plate locates in $z$-plane, at the same time, keeping the electric field line perpendicular to equipotential line after mapping. The field calculation becomes much easier in $\omega$-plane. And the field can be inversely transformed to $z$-plane through the following symbolic equations,

$$
\begin{aligned}
& E_{x}=\frac{2 V}{d} \frac{e^{u} \sin v}{1+2 e^{u} \cos v+e^{2 u}}, \\
& E_{y}=-\frac{2 V}{d} \frac{1+e^{u} \cos v}{1+2 e^{u} \cos v+e^{2 u}} .
\end{aligned}
$$

Since the surface charge density of the electrode is proportional to the field strength perpendicular to it, the capacitance can be calculated by

$$
C_{a c}=\frac{\varepsilon}{V} \int_{l} E_{y} d x
$$

where $l$ is the length of the electrode in $x$ direction.

However, this is unbounded when $l \rightarrow \infty$, which cannot represent the fringing capacitance of finite dimensions. Through approximation, the capacitance with electrode length equals to $l$ can be express as

$$
C \approx \varepsilon_{0} \frac{l}{d}+\frac{\varepsilon_{0}}{2 \pi} \ln \frac{2 \pi l}{d}
$$

where the first term is the capacitance caused by uniform field between two electrodes and the second term represents the fringing capacitance.

Moreover, for a 3D PP-Cap with side length $l$, side width $w$ and air gap distance $d$, the capacitance can be calculated as 
TABLE II.

DifFERENT CAPACITANCE Formula CONSIDERING FRINGING EFFECT

\begin{tabular}{|c|c|c|c|}
\hline Ref. & Auther & Year & Formula \\
\hline$[8]$ & Ignatowsky & 1932 & $C \approx C_{0}\left(1+\frac{2 d}{\pi R} \ln \frac{32 R}{e d}\right)$ \\
\hline$[9]$ & Cooke & 1958 & $C \approx C_{0}\left(1+\frac{2 d}{\pi R} \ln \frac{\pi e R}{d}\right)$ \\
\hline$[10]$ & Hutson & 1963 & $C \approx C_{0}\left(1+\frac{2 d}{\pi R} \ln \frac{8 \pi R}{d}\right)$ \\
\hline$[11]$ & Sloggett & 1986 & $C \approx C_{0}\left(1+\frac{2 d}{\pi R} \ln \frac{e \pi R}{d}\right)$ \\
\hline
\end{tabular}

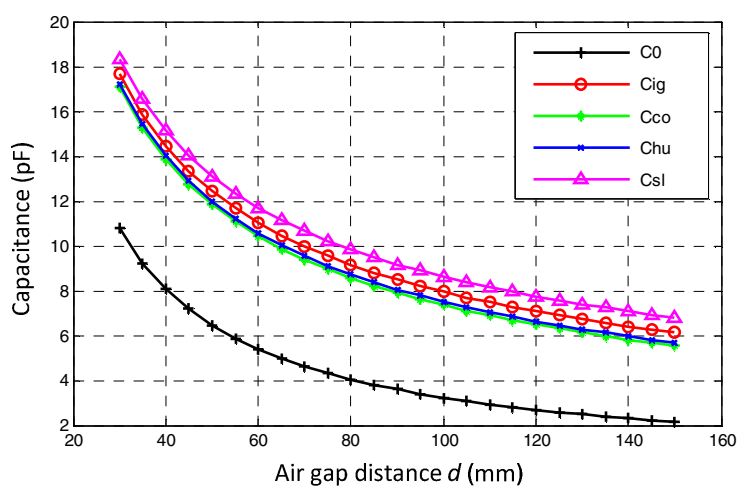

Figure 4. Comparison of calculated capacitance.

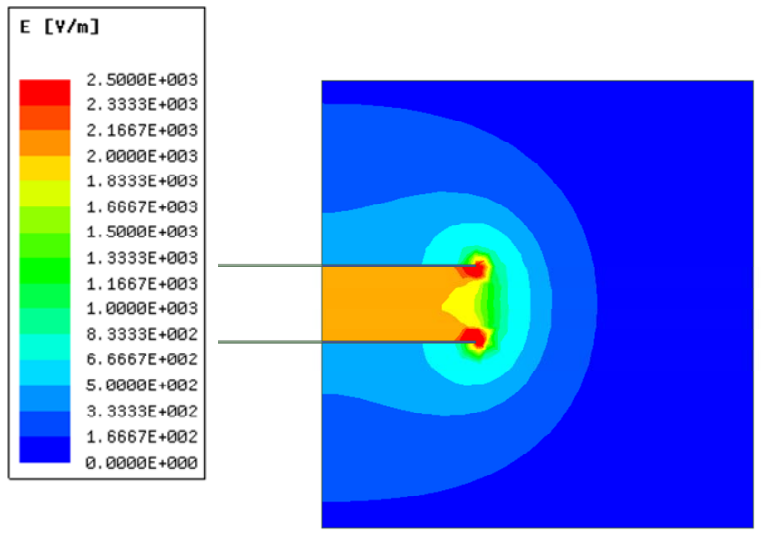

Figure 5. FEM Simulated fringing field of PP-Cap.

$$
C \approx \varepsilon_{0} \frac{l \cdot w}{d}+\frac{\varepsilon_{0} \cdot w}{2 \pi} \ln \frac{2 \pi l}{d} .
$$

The fringing electric field line and equipotential line can be plotted by solving (3) and (4) as shown in Fig. 3.

Depending on how the integration in (7) is approximated to make it convergent, different formulas have been proposed over the years, as summarized in Table II. In Table II, $C_{0}$ is the simplest capacitance calculated by $C_{0}=\varepsilon L^{2} / d$. $d$ is the air gap distance and $L$ is the side length for square plates. $R$ is theradius of the capacitor disc as a round disc capacitor are generally used to calculate fringing effect in these references.
For square capacitor, based on the principle of equal area, $R$ can approximately be replaced by

$$
R=\sqrt{\frac{L^{2}}{\pi}}
$$

To evaluate their accuracy in calculating the coupling capacitance for capacitive power transfer applications, the results solved by these formulas have been plotted in Fig. 4. The side length is defined as $200 \mathrm{~mm}$, and the air gap distance is variable of $30-150 \mathrm{~mm}$. From Fig. 4, the calculation results of the four formulas are all higher than $C_{0}$, as fringing effect will generally increase the coupling capacitance. The calculation result of Sloggett formula is the highest and about $2 \mathrm{pF}$ higher than the Cooke formula. The Ignatowsky and Hutson formulas are in between. When the air gap is $30 \mathrm{~mm}$, the calculation results are about $7 \mathrm{pF}$ higher than $C_{0}$ and the difference becomes smaller as air gap distance increases. When the air gap is $150 \mathrm{~mm}$, the calculation results are about $4 \mathrm{pF}$ higher than $C_{0}$. From Table II, it can be found that the formulas have similar structure, thus a general formula, with two parameters $\left(k_{1}\right.$ and $\left.k_{2}\right)$ to be determined, can be written as

$$
C \approx C_{0}\left(1+\frac{k_{1} d}{P} \ln \frac{k_{2} P}{d}\right) .
$$

And by FEM simulation and measurement, the two parameters can be determined. In this way, the general formula can give a more accurate calculation result in a certain range of side length and air gap distance.

\section{FEM SIMULATION}

FEM simulation is another way to obtain the capacitance with fringing effect considered. But simulation software is not available for everyone and the simulation process may take a long time if an accurate simulation result is wanted. In addition, it's not easy to add a time varying voltage excitation to the electrode in FEM simulation software. Thus, static voltage $+\mathrm{V}$ and $-\mathrm{V}$ are added to the electrodes as excitation.

Fig. 5 shows the simulated electric field around a parallel plate capacitor. The side length of $200 \mathrm{~mm}$ and the air gap distance of $50 \mathrm{~mm}$ are set, respectively. Comparing Fig. 3 with Fig.5, it can be seen that the calculated electric field line and equipotential line is similar but not exactly the same as the simulated result, especially in the middle of the outside center of the capacitor electrode. This is due to the assumption and approximation during the derivation of conformal mapping.

By changing the side length, airgap distance and repeating the simulation process, a set of capacitive versus side length and air gap distance can be obtained and they are plotted in Fig. 6. The simulation result is very close but a little bit higher than the calculation result of Sloggett formula.

\section{EXPERIMENTAL MEASUREMENT}

A measurement setup for the square parallel plate capacitor is implemented and shown in Fig. 7. The capacitor parameters are listed in Table III. To avoid been affected by the metal objects of the table, a hanging wooden plate is used to support the parallel plate capacitor. The measurement instrument is Agilent 4294A precision impedance analyzer with a bandwidth of $110 \mathrm{MHz}$ and the impedance analyzer is kept away from the capacitor to avoid affecting measurement 
TABLE III.

PARAMETERS OF EXPERIMENTAL SETUP

\begin{tabular}{|c|c|c|}
\hline Symbols & Description & Values \\
\hline$w$ & Width of the PP-Cap & $200 \mathrm{~mm}$ \\
\hline$l$ & Length of the PP-Cap & $200 \mathrm{~mm}$ \\
\hline$d$ & Air gap distance & $10-150 \mathrm{~mm}$ \\
\hline$f$ & Measurement frequency & $50 \mathrm{kHz}$ \\
\hline
\end{tabular}

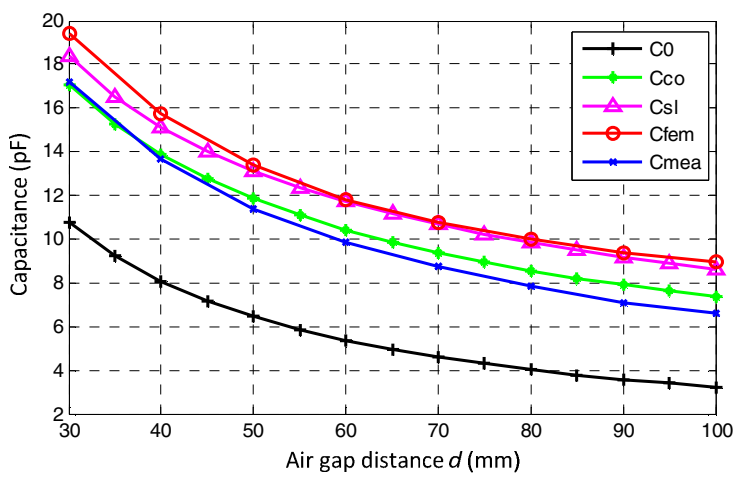

Figure 6. Comparison between measured results, simulated results and calculated results with corrected coefficients.

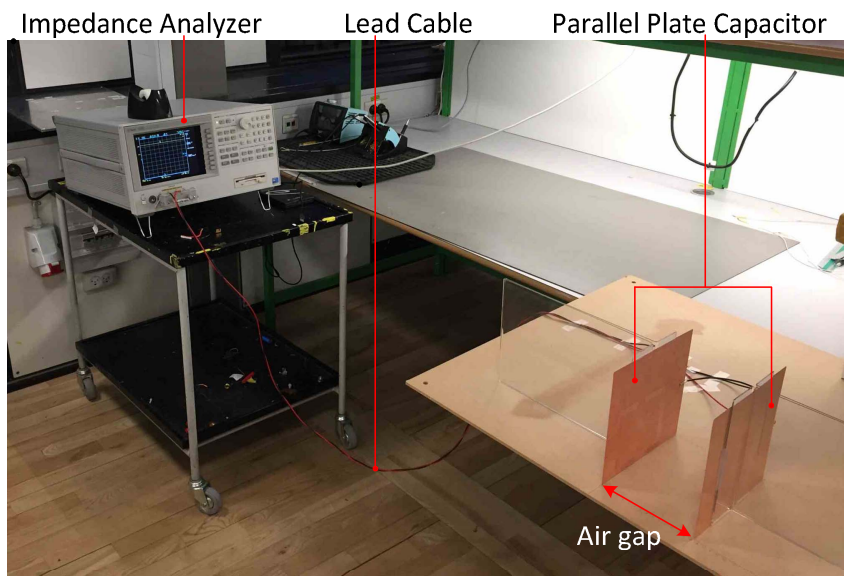

Figure 7. Parallel plate capacitor measurement setup.

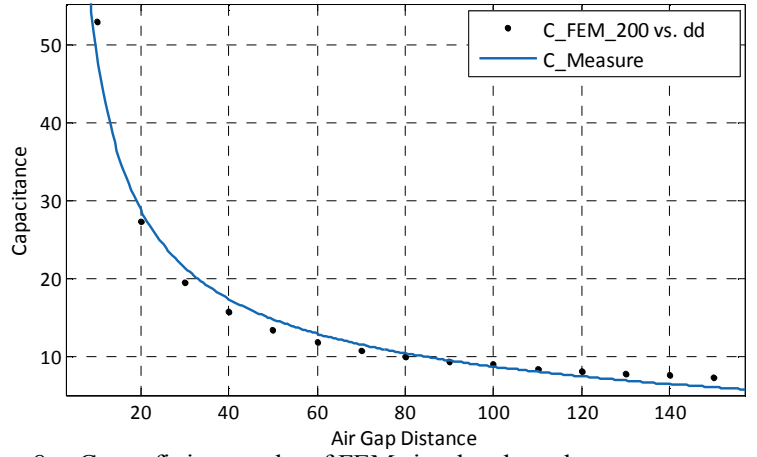

Figure 8. Curve fitting results of FEM simulated result.

results. The long measurement cable as well as the instrument itself is calibrated and fixed during the measurement process. The capacitance measurement results under different air gap distance are also plotted in Fig. 6. From Fig.6, the measurement result is higher than $C_{0}$ but lower than the simulation result and those calculations withfringing effect considered. Among the formulas given in Table II, the Cooke formula gives the closest calculation results to the measured value. Although the longer the air gap distance is, the larger the error. When the air gap distance is $30 \mathrm{~mm}$, the calculation result is $16.99 \mathrm{pF}$ and measurement result is $17.23 \mathrm{pF}$, which are very close to each other. When the air gap distance is 100 $\mathrm{mm}$, the calculated result is $7.24 \mathrm{pF}$ but the measured value is $6.63 \mathrm{pF}$. The simulation result is about $2.5 \mathrm{pF}$ higher that the measurement result throughout the process of air gap variation. One of the possible reasons is that in FEM simulation, there is no conductive objects around the capacitor, but in the measurement setup, although all the metal objects are kept away from the parallel plate capacitor, they may still have influence on the measurement results. Moreover, during the measurement process, it is found that the human body will also affect the measured capacitance and cause about $0.5 \mathrm{pF}$ capacitance drop if the human body is between the impedance analyzer and the capacitor.

Based on the analysis in the previous section, it can be concluded that the calculation of the capacitance of PP-Cap can be more precise by taken fringing effect into consideration. The capacitance can also be affected by nearby conductive objects like a human body, water and a metal box. The FEM simulation cannot give an accurate result if these conductive objects are not considered when building the simulation model.

Moreover, since the coupling capacitance is easily been affected by nearby objects. For the wireless power transfer system that using this kind of PP-Cap as transferring medium, the system stability and performance (like efficiency, soft switching condition) is better to be evaluated under a variable capacitance condition to ensure the robustness of the designed system. This phenomenon can also be used for foreign object detection, which is quite importance for wireless power transfer system. To obtain a more accurate formula to describe the measured capacitance, the weighted curve fitting method is used to determine the two coefficients $k_{1}$ and $k_{2}$ defined in (11), as shown in Fig.8. The calculated coefficients are $k_{1}=1.477$ and $k_{2}=1.55$ with $95 \%$ confidence bounds.

\section{CONCLUSION}

The capacitance of parallel plate capacitor considering fringing effect can be obtained using conformal mapping. For parallel plate capacitor used in high power capacitive power transfer application, where the side length is from $300 \mathrm{~mm}$ to $1000 \mathrm{~mm}$ and the distance range is from $10 \mathrm{~mm}$ to $180 \mathrm{~mm}$. The existing formulas cannot give an accurate calculation result. The essential reason is that conformal mapping can only be used for two-dimensional transformation, and moreover the electrical integration along the surface of capacitor plate is not convergent. Some approximation has been made during derivation of those formulas. A general formula with correction parameters is given in this paper. By using FEM simulation, measurement and curve fitting, the correction parameters are obtained for the specifications of capacitor used in capacitive power transfer application with the calculation error less than $3 \%$ compared to the measurement result. 


\section{REFERENCES}

[1] S. Oruganti, S. Heo, H. Ma, and F. Bien, "Wireless energy transferbased transceiver systems for power and/or high-data rate transmission through thick metal walls using sheet-like waveguides," Electron. Letters, vol. 50, no. 12, pp. 886-888, 2014.

[2] J. Dai, and D. C. Ludois, "A survey of wireless power transfer and a critical comparison of inductive and capacitive coupling for small gap applications," IEEE Trans. on Power Electron., vol. 30, no. 11, pp. 6017-6029, 2015.

[3] T. Kitabayashi, H. Funato, J. Haruna, Y. Tsuruta, and K. Yamaichi, "Loss analysis and power improvement of non-resonant type capacitive power transfer system with three-level operation," in Proc. IEEE ICPE-ECCE Asia, 2015, pp. 1678-1683.

[4] X. Chen, S. Yu, and Z. Zhang, "A Receiver-Controlled Coupler for Multiple Output Wireless Power Transfer Applications," IEEE Trans. Circuits and Systems I: Regular Papers, vol.66, no.11, pp.4542-4552, Nov. 2019.

[5] H. Zhang, F. Lu, H. Hofmann, W. Liu, and C. C. Mi, "Six-plate capacitive coupler to reduce electric field emission in large air-gap capacitive power transfer," IEEE Trans. on Power Electron., vol. 33, no. 1, pp. 665-675, 2017.
[6] H. Zhang, F. Lu, H. Hofmann, W. Liu, and C. C. Mi, "A large air-gap capacitive power transfer system with a 4-plate capacitive coupler structure for electric vehicle charging applications," in Proc. IEEE APEC., 2016, pp. 1726-1730.

[7] H. Zhang, F. Lu, H. Hofmann, W. Liu, and C. C. Mi, "An LCCompensated Electric Field Repeater for Long-Distance Capacitive Power Transfer," IEEE Trans. Ind. Appl., vol. 53, no. 5, pp. 49144922, 2017.

[8] B. Luo, R. Mai, Y. Chen, Y. Zhang, and Z. He, "A voltage stress optimization method of capacitive power transfer charging system," in Proc. IEEE APEC., 2017, pp. 1456-1461.

[9] W. Ignatowsky Academic Science URSS Trav. Inst. Steklof 3., vol.2, no.1, 1932.

[10] V. Hutson, "The circular plate condenser at small separations," in Proc. The Cambridge Philosophical Society (Mathematical)., vol. 59, no.1, pp. 211-224, 1963

[11] B. D. Hughes, "Comment on the potential due to a circular parallel plate capacitor," Citeseer, 1983.

[12] G. J. Sloggett, N. G. Barton, and S. J. Spencer, "Fringing fields in disc capacitors," Journal of Physics A: Mathematical and General., vol.19, no.14, pp. 2725-2736, 1986. 